CASE REPORT

\title{
Endometrial coccidiosis
}

\section{R D de Otazu, L García-Nieto, E Izaguirre-Gondra, E Mayayo, S Ciani, F F Nogales}

J Clin Pathol 2004;57:1 104-1105. doi: 10.1136/jcp.2004.018283

This report describes a case of granulomatous endometritis caused by coccidiosis in an immunologically uncompromised 63 year old patient. The glandular epithelium of the endometrium contained numerous intracytoplasmic cysts, corresponding to periodic acid Schiff positive and methenamine silver negative sporoblasts. The endometrial glands revealed reactive phenomena, such as eosinophilic and squamous glandular metaplasia and intraluminal desquamation. Non-necrotising epithelioid granulomata, lacking the presence of parasites, were present in the stroma. Although not detected in the stool examination, the organisms were probably Isospora belli. There was no evidence of other foci of the disease. Coccidiosis should be differentiated from the more commonly occurring coccidiomycosis.

G ranulomatous endometritis other than as a result of Mycobacterium tuberculosis ${ }^{1}$ is rare and related to a variety of aetiological agents, such as bacteria, unicellular or multicellular parasites, worms, ${ }^{2}$ fungi, ${ }^{3}$ immunological responses, and even postraumatic phenomena after endometrial resection. ${ }^{4}$ Many such cases of endometritis occur in patients with immunodeficiency.

This report presents the first case of coccidiosis, a protozoan disease causing chronic inflammation, in the endometrium of an otherwise immunologically uncompromised patient.

\section{CASE HISTORY}

This 63 year old Spanish housewife presented with metrorrhagia; an aspiration endometrial biopsy performed on admission showed a granulomatous endometritis. In the ultrasound examination a solid left adnexal mass was found. She underwent total abdominal hysterectomy with bilateral salpingo-oophorectomy and biopsy of enlarged iliac lymph nodes. Macroscopically, the left $12 \mathrm{~cm}$ ovarian mass was solid, homogeneous, white, and fasciculated; the remaining female genital tract showed involutive features. Microscopically, the adnexal tumour corresponded to an ovarian fibrothecoma, and the atrophic endometrium showed patches of chronic inflammation, with typical non-necrotising epithelioid granulomata, and no microorganisms or inclusions were present (fig 1). The endometrial glands had undergone abundant intraluminal desquamation. A striking feature was the presence in the parietal and desquamated epithelial cells of numerous intracytoplasmic cysts (figs 2, 3); these cysts measured 20-30 $\mu \mathrm{m}$ and were filled with periodic acid Schiff positive and Grocott's methenamine silver negative organisms, or with a fine basophilic granular material. These formations were identified as the oocysts containing sporoblasts of coccidiosis. All parasitised glandular epithelia had undergone eosinophilic changes; there were also isolated areas of glandular squamous metaplasia.

After diagnosis, a thorough examination of the patient's past history failed to reveal concomitant episodes of diarrhoea and only sporadic contact with domestic animals was documented. Stool examination was negative. Specific investigations for human immunodeficiency virus, diabetes, and other causes of immunodeficiency all proved negative. Serology for coccidiosis, blastomycosis, and histoplasma were also negative. Mild eosinophilia persisted in the immediate postoperative period. The patient is well with no evidence of disease four years after diagnosis.

\section{DISCUSSION}

Human coccidiosis is a rare protozoan disease caused by four different types of sporozoans (cyclospora, cryptospora, sarcocystis, and isospora); of these, Isospora belli is the most frequent in humans. ${ }^{5}$ In the absence of a definite identification in the stool examination, endometritis in our patient was thought to be caused by such an organism, taking into account the size $(20-30 \mu \mathrm{m})$ and morphology of the cysts, although they may be difficult to differentiate morphologically from those of sarcocystis. Isospora belli preferentially involves the digestive tract of immunodeficient patients, who may acquire the infective oocysts orally.

\section{"Endometrial coccidiosis should be differentiated from the more commonly occurring coccidiomycosis, an inflamma- tion caused by the fungus Coccidioides immitis, which can occasionally involve the endometrium"}

Although coccidiosis may also affect other organs outside the intestine such as the spleen, liver, and lymph nodes, ${ }^{6}$ this is the first time that it has been reported in the endometrium. The pathogenesis of the endometritis in our patient remains obscure because she had not had diarrhoea and had no signs of immunodeficiency. The localised character of the inflammation present in this patient is similar to intestinal

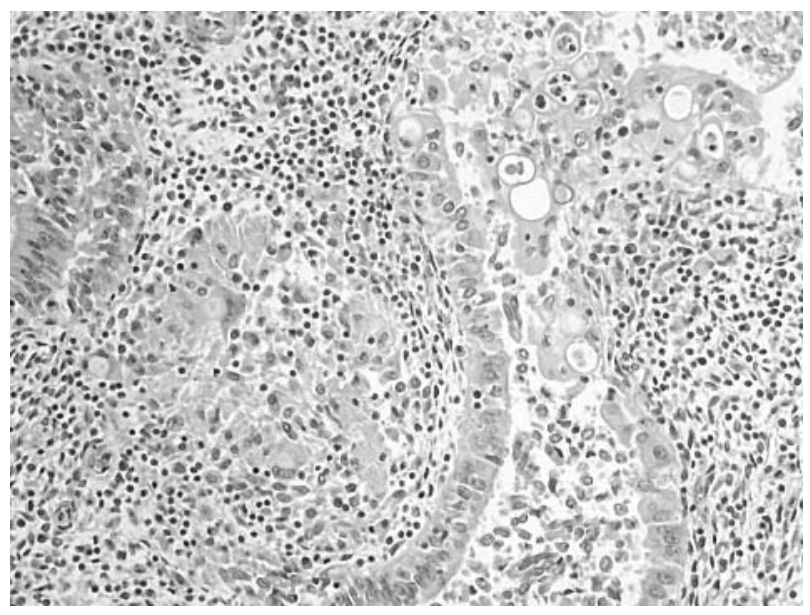

Figure 1 Endometrium showing epithelioid granuloma and gland with numerous intracytoplasmic cysts and desquamated cells in the lumen. 


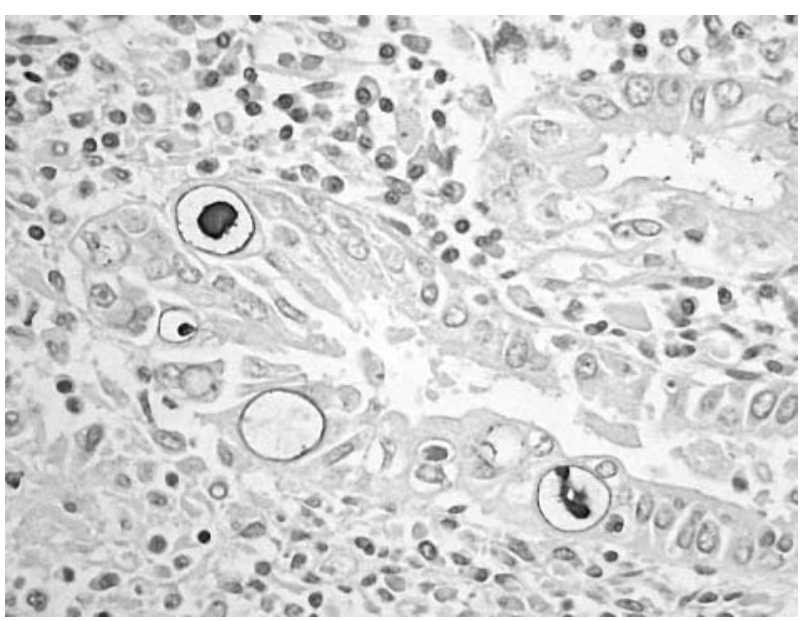

Figure 2 Glandular epithelial cysts containing sporoblasts.

coccidiosis occurring in immunologically uncompromised patients, where it is invariably a self limiting process. ${ }^{5}$ The focal nature of coccidiosis in our patient is exemplified by the absence of parasites or granulomata in the iliac lymph nodes removed at surgery. In this case, it is likely that the oocysts, possibly acquired from domestic animals, may have reached the endometrium from the lower female genital tract. In contrast, endometrial tuberculosis is in most cases secondary to descending infection from salpingitis. ${ }^{1}$

Histopathologically, the endometrium showed the characteristic involvement of coccidiosis, revealing a glandular epithelium laden with intracytoplasmic cysts that corresponded to oocysts and sporoblasts. The presence of the protozoan elicited an eosinophilic epithelial change similar to that seen in female genital tuberculosis in the vicinity of granulomata. ${ }^{1}$ In addition, chronic inflammation also induced foci of squamous metaplasia in the glands, as is often seen in chronic endometritis.

Endometrial coccidiosis should be differentiated from the more commonly occurring coccidiomycosis, an inflammation caused by the fungus Coccidioides immitis, which can occasionally involve the endometrium. ${ }^{3}$ Although both exhibit the presence of cysts, those occurring in coccidiomycosis are usually larger and filled with methenamine silver positive endospores. Moreover, coccidiomycosis does not usually involve the epithelium but preferentially colonises stromal macrophages. Malacoplakia, a chronic inflammatory condition caused by bacterial infection and leucocyte functional impairment, may involve the endometrium, ${ }^{7}$ and also presents with epithelioid granulomata. In this condition, however, macrophages show the characteristic Michaelis Gutmann bodies, a feature absent in coccidiosis.

It is proposed that coccidiosis should be taken into account in the differential diagnosis of granulomatous endometritis.

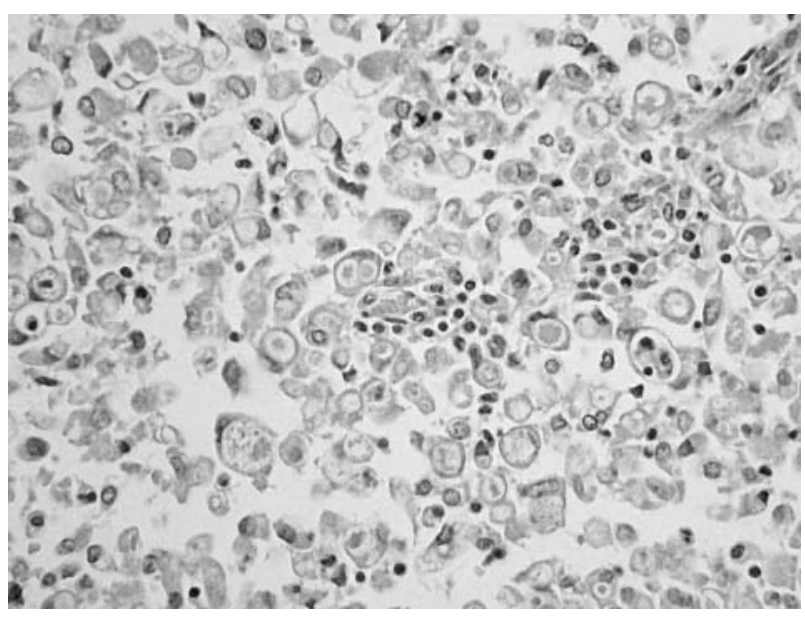

Figure 3 Desquamated epithelium with numerous sporoblasts.

\section{Take home messages}

- We report the first case of coccidiosis, a protozoan disease causing chronic inflammation, in the endometrium of an otherwise immunologically uncompromised patient

- Coccidiosis should be taken into account in the differential diagnosis of granulomatous endometritis

\section{Authors' affiliations}

R D de Otazu, L García-Nieto, E Izaguirre-Gondra, Department of Pathology, Hospital Txagorritxu, 01010 Alava, Spain

E Mayayo, University of Tarragona, 43006 Tarragona, Spain S Ciani, F F Nogales, University Hospital, 18012 Granada, Spain

Correspondence to: Dr F F Nogales, Departamento de Anatomía Patológica, Facultad de Medicina, 18012 Granada, Spain; fnogales@ ugr.es

Accepted for publication 20 May 2004

\section{REFERENCES}

1 Nogales-Ortiz F, Tarancon I, Nogales FF Jr. The pathology of female genital tuberculosis. A 31-year study of 1436 cases. Obstet Gynecol 1979;53:422-8.

2 Mayayo E, Mestres M, Sarmiento J, et al. Pelvic oxyuriasis. Acta Obstet Gynecol Scand 1986;65:805-6.

3 Bylund DJ, Nanfro JJ, Marsh WL Jr. Coccidioidomycosis of the female genital tract. Arch Pathol Lab Med 1986;110:232-5.

4 Smart PJ, Hetherington JF. Postoperative uterine granulomata following endometrial resection. Pathology 1995;27:209-11.

5 Goodgame RW. Understanding intestinal spore-forming protozoa: cryptosporidia, microsporidia, isospora, and cyclospora. Ann Intern Med 1996; 124:429-41.

6 Michiels JF, Hofman P, Bernard E, et al. Intestinal and extraintestinal Isospora belli infection in an AIDS patient. A second case report. Pathol Res Pract 1994; 190:1089-93.

7 Chou SC, Wang JS, Tseng HH. Malacoplakia of the ovary, fallopian tube and uterus: a case associated with diabetes mellitus. Pathol Int 2002;52:789-93. 